\title{
Thermal Reactions of Blood Vessels in Vascular Stroke and Heatstroke
}

\author{
Olav Thulesius
}

Department of Clinical Physiology, Faculty of Medicine, University Hospital Linköping, Linköping, Sweden

\begin{abstract}
Key Words
Heatstroke - Exertional heatstroke · Ischemic stroke • Hyperthermia - Hypothermia - Thermal vasomotor responses $\cdot$ Carotid artery cooling $\cdot$ Neuroprotection
\end{abstract}

\begin{abstract}
Research on the pathophysiology and treatment of brain damage with special focus on thermal vascular responses is the subject of this minireview. Interruption of cerebral blood supply by vascular obstruction, temporary cardiac arrest or hyperthermia causes a sudden attack of vascular stroke or heatstroke with serious consequences. It may not induce immediate cell death, but can precipitate a complex biochemical cascade leading to a delayed neuronal loss. When testing thermal vasomotor responses by stepwise cooling of isolated carotid arteries, a temperature-proportional dilatation was observed while heating induced the opposite response: a marked vasoconstriction. General hyperthermia with an increased oxygen demand combined with a reduction of blood supply therefore is a serious consequence. At the cellular level an important mechanism involving hyperthermia is the temperature-dependent regulation of $\mathrm{K}^{+}$ channel tone of vascular smooth muscle. Further, their inhibition through temperature elevation causes vasoconstriction. In heatstroke, which can induce platelet aggregation and the release of the vasoconstrictor sero-
\end{abstract}

tonin, arterial cooling attenuates this response. General hypothermia is induced to prevent or attenuate neurological damage in stroke. The procedure is not without serious side effects. Therefore, rapid institution of selective brain cooling has been considered in adults and in infants with postpartum encephalopathy.

Copyright (C) 2006 S. Karger AG, Basel

\section{Heatstroke}

Heatstroke (HS) is a disorder with a core body temperature $>40^{\circ} \mathrm{C}$. Two forms of HS are recognized, classic HS, usually occurring in elderly persons, and exertional HS in physically active younger individuals. It occurs in a hot environment and can be triggered by strenuous exercise. Body temperature regulation involves a homeostatic balance between heat production and dissipation. Sympathetic agents such as amphetamine, ephedrine and ecstasy can disrupt this balance and as a result produce potentially life-threatening hyperthermia. HS is a complex disorder of thermoregulation with multiple organ dysfunction, arterial hypotension, renal failure, disseminated intravascular coagulation, rhabdomyolysis, acidbase and electrolyte disturbances. It is a medical emergency with an optimal prognosis when diagnosed early and promptly managed with cooling, fluid and electrolyte replacement [1].

\section{KARGER}

Fax +4161306 1234

E-Mail karger@karger.ch

www.karger.com
(ㄷ) 2006 S. Karger AG, Basel

$1011-7571 / 06 / 0154-0316 \$ 23.50 / 0$

Accessible online at:

www.karger.com/mpp
Prof. Olav Thulesius, MD, PhD

Stormvägen 31

SE-58274 Linköping (Sweden)

Tel./Fax +4613273 632,E-Mail olav.thulesius@telia.com 
Investigations on HS in Kuwait started in the 1980s at the Faculty of Medicine. Interest in the subject was initiated when the yearly pilgrimage to Mecca coincided with the hot season. In 1981, 480 people were treated for HS in the emergency clinics and hospitals in Saudi Arabia and the following year with 2 million participants it was estimated that there were 1,119 cases of HS and 8,000 suffered heat exhaustion [2, 3]. But HS is not limited to countries with a tropical climate. The incidence varies seasonally and can be up to 250 cases per 100,000 population. There have been serious accidents in urban areas in the United States with an incidence of up to 27 cases per 100,000 population mostly affecting the elderly since the ability to make physiological adjustments when exposed to high temperatures decreases with age [4]. High mortality observed in HS is secondary to multiorgan dysfunction. Aggressive measures to lower body temperature combined with other supportive therapy substantially reduces mortality.

A chief feature of HS, as compared to febrile conditions at equal temperature, is a reduced cerebral blood flow with ischemia and the onset of HS being arbitrarily defined as when cerebral blood flow begins to decrease from its peak level. High levels of CPK (>1,000 IU/l), metabolic acidosis, and elevated liver enzymes are predictive. Severe cases with multiple organ dysfunction have elevation of cytokines and chemokines and increased levels of serotonin and dopamine in the hypothalamus and corpus striatum $[5,6]$. Reduced splanchnic blood flow and hyperthermia during exercise-heat stress can produce gastrointestinal barrier dysfunction with increased permeability. This may allow endotoxin to enter the internal environment, causing local and systemic immune responses which may be involved in the cause of HS. Countermeasures can reduce gastrointestinal permeability and possibly occurrence and outcome [7].

Heat stress can induce blood clotting since it directly activates platelets and this also leads to a reduced thrombocyte count with purpura and bleeding, characteristic of consumption coagulopathy $[8,9]$. Therefore, in severe cases, aggressive intensive care treatment with forced diuresis and endovascular cooling may be necessary to combat HS [10].

\section{Thermal Vascular Responses}

Because adequate blood supply to vital organs is of utmost importance, extensive investigations into the thermal responses of blood vessels were performed by

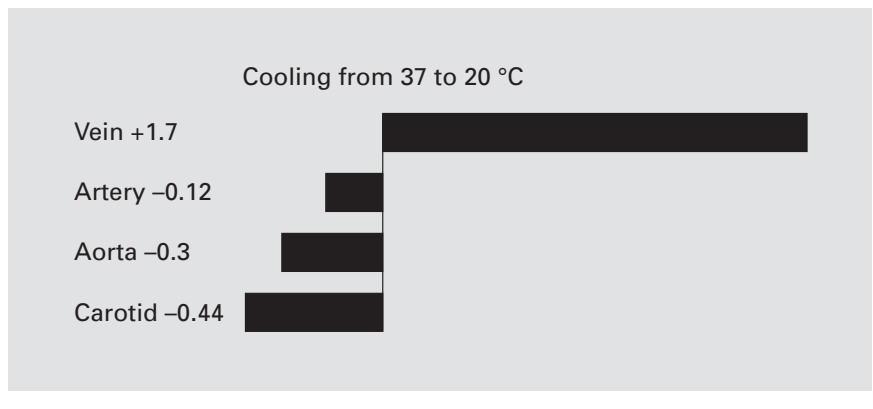

Fig. 1. Effect of hypothermia on superficial sheep extremity veins and deep arteries, rat aorta and rabbit carotid artery specimens (tension in grams per gram tissue weight).

recording the tension of vascular rings as indicators of vasodilatation or vasoconstriction in thermostated organ baths. Using specimens of various types of blood vessels from different species (human, sheep, rabbit and rat), hyper- and hypothermia induces significant changes of smooth muscle tone. These reactions were found to be independent of neurogenic control.

There is a marked regional difference in the thermal responses of arteries and veins depending on the functional role of the vessel in extremities or at a vital central location of the body. Skin blood flow in man and homeothermic animals is reduced when exposed to a cold environment. This response is usually explained to be due to a reflexogenic increase in sympathetic vasoconstrictor tone if cooling threatens to reduce body temperature. Local cooling increases tone in superficial cutaneous extremity veins, proportional to cooling temperature and independent of alpha-adrenergic innervation. The concomitant deep artery of the same region displayed the opposite reaction: a cooling-induced vasodilatation, also not mediated by nervous influence. Locally induced cold vasoconstriction is difficult to reconcile with our knowledge about the positive temperature-dependent process of nerve conduction and transmitter release, but the response is myogenic and due to transcellular ion translocation [11]. A summary of the effect of hyperthermia on superficial extremity veins and deep arteries, rat aorta and rabbit carotid artery specimens is presented in figure 1 .

When investigating renal arteries, it was detected that the 5-HT-induced vasoconstriction was potentiated by heating [12]. This finding is of relevance to HS since heatinduced thrombocyte aggregation leads to the release of serotonin and therefore may be one of the factors inducing kidney failure. Dose-response curves of 5-HT contractions are shown in figure 2. Further investigations cen- 


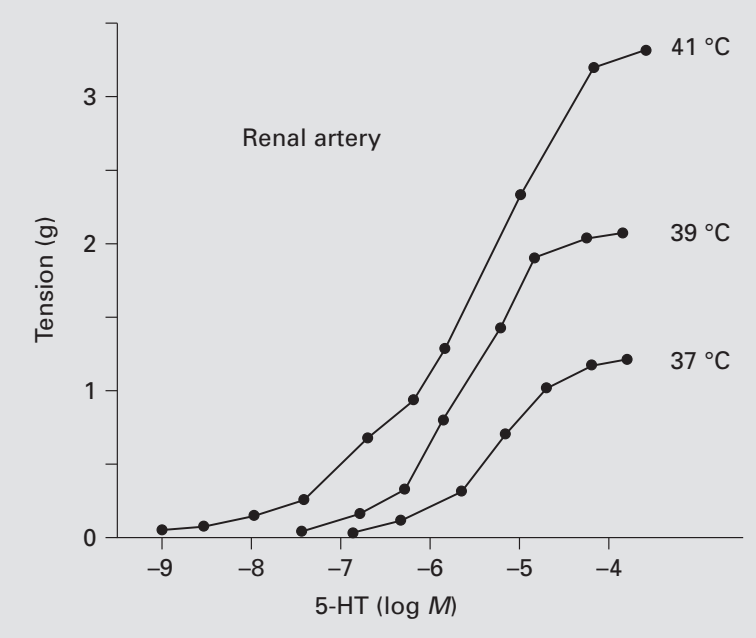

Fig. 2. Dose-response curves to 5-HT of isolated sheep renal artery specimens. Elevating the bath temperature resulted in an increased contractile response.

tered on the aorta, pulmonary artery and vena cava, which displayed clear vasodilatation when exposed to cooling. Dilatation was directly proportional to the drop in temperature and not due to endothelium-dependent NO release or to other vasodilator substances. The reaction could be elicited at basal tone and after precontraction with noradrenaline and $\mathrm{KCl}$. Failure of the neuron blocker tetrodotoxin to attenuate the reaction implies that it is not a neurogenic response [13].

\section{Carotid Artery}

Stepwise cooling of isolated rings of rabbit carotid arteries induced a temperature-proportional dilatation [14]. On the other hand, heating or hyperthermia had the opposite effect: a marked vasoconstriction of the carotid artery, and it can be assumed that this reaction may contribute to a decrease in cerebral blood flow in HS [15].

Thermal reactions of the carotid artery as summarized in figure 3 are the most relevant from the point of view of hyperthermia because a decline in cerebral blood flow is the salient feature of HS. Cooling favors vasodilatation, a fact that could play a role when utilizing therapeutic cooling if applied to the neck. A general conclusion of these experiments is that anatomically blood vessels such as arteries and veins may display a marked regional difference

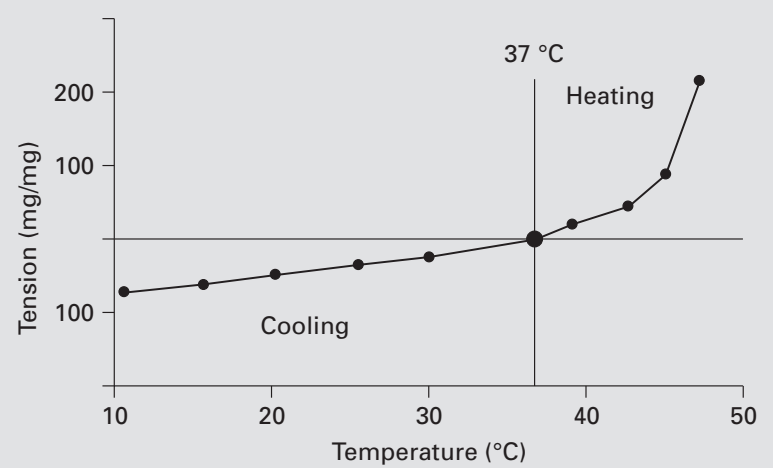

Fig. 3. Effects of cooling and heating on the contractile response of rabbit carotid artery specimens. Note the potentiated response during hyper- and hypothermia.

in responses depending on local function. This, as illustrated in figure 1 , shows that the most pronounced thermal vasoconstrictor response occurred in a peripheral extremity vein while on the arterial side a significant hypothermic relaxation was obtained in the carotid artery.

In order to investigate the underlying mechanism of the thermal responses in the carotid artery at the cellular level, blocking experiments were performed that revealed a critical involvement of potassium channels $\left(\mathrm{K}^{+}\right.$channels), important regulators of smooth muscle function and hence vascular diameter. Activation of these induces a major vasodilatory mechanism through membrane hyperpolarization, while their inhibition causes vasoconstriction. Four distinct types of $\mathrm{K}^{+}$channels have been identified in arterial smooth muscle [16, 17]. It was found that heating-induced contractions were potentiated by the K-channel inhibitors tetraethylammonium, $\mathrm{BaCl}_{2}$, charybdotoxin and the $\mathrm{Na}^{+} / \mathrm{K}^{+}$ATPase inhibitor ouabain. On the other hand, levcromakalim, a $\mathrm{K}^{+}$channel activator, reduced heating-induced contractions [18]. Consequently, $\mathrm{K}^{+}$channel openers like levcromakalim may be useful drug candidates to treat delayed cerebral vasospasm after cerebral hemorrhage, as recently shown [19]. 


\section{Treatment}

Heat illness prevention is the first important step by excluding risk factors such as lack of acclimatization, sleep deprivation and certain medications (e.g. diuretics). Proper hydration is essential since the need for evaporative heat loss is increased due to fever and exercise and environmental heat transfer. Core body temperature has been shown to rise $2^{\circ} \mathrm{C}$ for every $1 \%$ of body weight lost to dehydration [20]. The main predictor of outcome in severe exertional HS is the duration and degree of hyperthermia.

Interruption of cerebral blood flow leads to increased intracellular calcium and sodium concentration followed by generation of free radicals and initiation of apoptosis. The process is associated with edema formation and compression of adjacent nonischemic neurons. Each step in this complex, interdependent series of events offers a potential point to intervene and prevent neuronal death. Although many human trials in acute stroke therapy have had disappointing results, new promising procedures are in the pipeline, including various types of hypothermia and free-radical inhibitors [21].

Experimental hypothermia inhibits many steps in the biochemical cascade that produces severe brain injury after hypoxia-ischemia. This is in contrast to pharmacological agents, which tend to target only one step in the process. A characteristic feature of HS is a coagulopathy with hyperaggregability of platelets. This mechanism, once started, is unaffected by cooling but can be prevented by early administration of aspirin or clopidogrel. Oxidative stress generated during HS due to hydroxyl radicals has been implicated in brain damage and it has been shown that pretreatment with $\alpha$-tocopherol (vitamin E or derivatives such as magnol) can attenuate damage [22].

In a study with mild hypothermia $\left(31-33^{\circ} \mathrm{C}\right)$, blood flow was measured in patients undergoing coronary artery bypass surgery. Arterial pressure and blood flow were recorded in the middle cerebral artery to calculate cerebral vascular resistance which showed preservation of autoregulation. Autoregulation of cerebral blood flow is a hallmark of the cerebral circulation and provides adequate nutritive blood flow despite fluctuations in arterial pressure. This means that with cooling there was no reduction in flow even with arterial hypotension [23].

General hypothermia can be administered by cold water immersion, cooling blankets or endovascular cooling. These procedures can effectively lower body temperature, but they are not without serious risks as shown in a study where the most frequent complications were pul- monary infection, arterial hypotension, bradycardia, arrhythmia, and thrombocytopenia [24].

The high incidence of stroke in Saudi Arabia prompted the development of an evaporative method of fast body cooling by spraying patients with water at $15^{\circ} \mathrm{C}$ and warm air to prevent shivering. This method is superior to immersion in ice-cold water since it prevents shivering. Patients were admitted to special Heat Stroke Treatment Centers, equipped with these cooling units [25].

\section{Selective Brain Cooling}

There are several advantages of locally applied hypothermia or selective brain cooling (SBC), such as fast administration with ice pads placed around the neck followed by a cooling collar at the neck, a head-cooling cap or facial cooling during ambulance transport. A cooling collar was designed that can be perfused by glycol, the temperature of which can be adjusted by a thermocircular system. Cooling of the neck may reduce temperature of blood reaching the brain and induce carotid artery vasodilatation [26]. The application of facial fanning can directly lower brain temperature by inward blood flow through angularis oculi veins. Normally, flow is directed from brain to face but it can also turn in the opposite direction from the face to the cavernous sinus [27]. Facial cooling and cold upper respiratory airflow has been used therapeutically in brain-injured patients to induce SBC [28]. A recent large multicenter randomized trial of head cooling for neonatal encephalopathy strongly suggest that prolonged cerebral hypothermia is both generally safe - at least in an intensive care setting - and can improve intact survival up to 18 months of age [29]. Another study in term infants with hypoxic-ischemic encephalopathy SBC was commenced within $6 \mathrm{~h}$ of birth and continued for 48-72 h. Here a decrease in the incidence of severe cortical lesions was seen [30].

Under normal conditions, the brain and core temperatures are approximately equal, but in some diseased states, brain temperature is higher in head-injured patients [31]. An experimental study to evaluate the effect of SBC showed that cortical cerebral blood flow, as measured by laser-Doppler flowmetry, increased to $215 \%$ of baseline at a cortical brain temperature of $30.9^{\circ} \mathrm{C}$ and a rectal temperature of $37.5^{\circ} \mathrm{C}$. During rewarming, as brain temperature increased, blood flow decreased. The cerebral vasodilatory response to hypothermia may explain its protective effects during and after cerebral ischemia [32]. 
Therefore, it seems reasonable to apply cold noninvasively by transcutaneous cooling of the head and neck close to the carotid and vertebral arteries, thereby inducing vasodilatation and transport of cooled blood to the brain. It remains to be seen if CBF can be increased by a localized cooling procedure and if transfer of cooled blood to the brain induces an in vivo dilatation of downstream arterioles. This process should have a twofold beneficial effect: selective cooling of brain tissue and vasodilatation.

The principle of the cooling collar is a temperaturecontrolled device that can induce cooling of blood reaching the CNS through the carotid and vertebral arteries. The collar provides an isolated passageway for chilled fluid to circulate around the skin of the neck, thus absorbing heat by conduction from adjacent blood vessels. The technique should be useful in the rapid initial treatment of HS. A theoretical model of flow dynamics in the carotid artery revealed that cooling of arterial blood can yield a temperature that is $1.10^{\circ} \mathrm{C}$ lower than body core temperature when the neck surface is cooled to $0^{\circ} \mathrm{C}$ with ice [33].

A cooling helmet for the treatment of stroke has been developed by using NASA spin-off technology. Its effectiveness in lowering brain temperature in patients with severe stroke or head injury was examined in patients and controls. Brain temperatures were continuously monitored for $72 \mathrm{~h}$ and compared with core temperature. There was an initial rapid and selective brain cooling and it maintained a significant temperature gradient between brain and core, minimizing systemic complications. On average, $1.84^{\circ} \mathrm{C}$ of brain temperature reduction was observed within $1 \mathrm{~h}$ to achieve a brain temperature lower than $34^{\circ} \mathrm{C}$ and $6.7 \mathrm{~h}$ before systemic hypothermia $\left(<36^{\circ} \mathrm{C}\right)$ occurred [34]. Recently another microcontroller cooling helmet for SBC has been proposed [35]. The cooling collar may be combined with a cooling helmet.

\section{Conclusions}

Studies of thermal reactions of blood vessels show that cooling of carotid arteries induces vasodilatation and heating vasoconstriction. In therapeutic terms, related to vascular stroke and HS, this means that selective hypothermia applied to the head and neck region may increase cerebral perfusion. In HS, which can induce platelet aggregation and the release of the vasoconstrictor serotonin, arterial cooling attenuates this response.

\section{References}

1 Bouchama A, Knochel JP: Heat stroke. N Engl J Med 2002;346:1978-1988.

2 Thulesius O: Heat stroke or siriasis and the effect on blood vessels. Forskning Praktik 1986; 18:48-50.

3 Ghaznavi HI, Ibrahim MA: Heat stroke and heat exhaustion in pilgrims performing the $\mathrm{Haj}$ (annual pilgrimage) in Saudi Arabia. Ann Saudi Med 1987;7:323-326.

-4 Jones TS, Liang AP, Kilbourne EM, et al: Morbidity and mortality associated with the July 1980 heat wave in St. Louis and Kansas City, Mo. JAMA 1982;247:3327-3331.

-5 Lin MT, Lin SZ: Cerebral ischemia is the main cause for the onset of heat stroke syndrome in rabbits. Experientia 1992;15:225-227.

6 Lin MT: Heatstroke-induced cerebral ischemia and neuronal damage: involvement of cytokines and monoamines. Ann NY Acad Sci 1997; 13:572-580

-7 Lambert GP: Role of gastrointestinal permeability in exertional heatstroke. Exerc Sport Sci Rev 2004;32:185-190.

-8 Mohanty D, Gomez J, Mustafa KY, Khogali M, Das KC: Pathophysiology of bleeding in heat stress: an experimental study in sheep. Exp Hematol 1997;25:615-619.
Gader AM, Al-Mashhadani SA, Al-Harthy SS Direct activation of platelets by heat is the possible trigger of the coagulopathy of heat stroke. Br J Haematol 1990; 74:86-92.

-10 Broessner G, Beer R, Franz G, Lackner P, Engelhardt $\mathrm{K}$, Brenneis $\mathrm{C}$, Pfausler B, Schmutzhard E: Case report: severe heat stroke with multiple organ dysfunction - a novel intravascular treatment approach. Crit Care 2005;9: R498-501.

11 Thulesius O, Yousif M: Na+, K+-ATPase inhibition, a new mechanism for cold-induced vasoconstriction in cutaneous veins. Acta Physiol Scand 1990;141:127-128

12 Elkhawad AO, Thulesius O, Khoghali M: Vascular effects of hyperthermia on isolated blood vessels. Gen Pharmacol 1983;14:69-71.

13 Mustafa ES, Thulesius O: Cooling is a potent vasodilator of deep vessels in the rat. Can $\mathrm{J}$ Physiol Pharmacol 2001;19:899-904.

14 Mustafa S, Thulesius O: Cooling-induced carotid artery dilatation, an experimental study in isolated vessels. Stroke 2002;33:256-260.

15 Mustafa S, Thulesius O: Hyperthermia-induced vasoconstriction of the carotid artery, a possible causative factor of heatstroke. J Appl Physiol 2004;96:1875-1879.
16 Gluais Standen NB, Quayle JM: K+ channel modulation in arterial smooth muscle. Acta Physiol Scand 1998;164:549-557.

17 Faraci FM, Heistad DD: Regulation of the cerebral circulation: role of endothelium and potassium channels. Physiol Rev 1998;78:5397.

18 Mustafa S, Thulesius O: Hyperthermia-induced vasoconstriction of the carotid artery and the role of potassium channels. J Stroke Cerebrovasc Dis 2005; 14:122-126.

19 Sugai K, Yanagisawa T, Motohashi O, Suzuki $\mathrm{M}$, Yoshimoto T: Levcromakalim decreases cytoplasmic $\mathrm{Ca} 2+$ and vascular tone in basilar artery of SAH model dogs. J Cardiovasc Pharmacol 1999;33:868-875.

20 Coris EE, Ramirez AM, Van Durme DJ: Heat illness in athletes: the dangerous combination of heat, humidity and exercise. Sports Med 2004;34:9-16.

21 Smith WS: Pathophysiology of focal cerebral ischemia: a therapeutic perspective. J Vasc Interv Radiol 2004;15:S3-12.

22 Niu KC, Lin KC, Yang CY, Lin MT: Protective effects of alpha-tocopherol and mannitol in both circulatory shock and cerebral ischaemic injury in rat heatstroke. Clin Exp Pharmacol Physiol 2003;30:745-751. 
-23 Preisman S, Marks R, Nahtomi-Shick O, Sidi A: Preservation of static and dynamic cerebral autoregulation after mild hypothermic cardiopulmonary bypass. Br J Anaesth 2005;95:207211.

24 Georgiadis D, Schwarz S, Kollmar R, Schwab $\mathrm{S}$ : Endovascular cooling for moderate hypothermia in patients with acute stroke: first results of a novel approach. Stroke 2001;32: 2550-2553.

25 Weiner JS, Khogeli M: A physiological bodycooling unit for treatment of heat stroke. Lancet 1980;1:507-509.

26 Thulesius O: The effect of hypothermia and its potential in the treatment of brain injury. Arbete \& Halsa 2002;13:55-60.

27 Caputa M, Perrin G, Cabanac M: Reversal of human ophthalmic vein blood flow: selective cooling of the brain. CR Acad Sci 1976;30: 1011-1014.
28 Andrews PJ, Harris B, Murray GD: Randomized controlled trial of effects of the airflow through the upper respiratory tract of intubated brain-injured patients on brain temperature and selective brain cooling. Br J Anaesth 2005; 94:330-335.

29 Gluckman PD, Wyatt JS, Azzopardi D, Ballard R, Edwards AD, Ferriero DM, Polin RA, Robertson CM, Thoresen M, Whitelaw A, Gunn AJ: Selective head cooling with mild systemic hypothermia after neonatal encephalopathy: multicentre randomised trial. Lancet 2005;19-25;365:663-670.

30 Rutherford MA, Azzopardi D, Whitelaw A, Cowan F, Renowden S, Edwards AD, Thoresen M: Mild hypothermia and the distribution of cerebral lesions in neonates with hypoxicischemic encephalopathy. Pediatrics 2005; 116:1001-1006.
1 Rumana CS, Gospinath SP: Brain temperature exceeds systemic temperature in head-injured patients. Crit Care Med 1998;26:562-567.

32 Kuluz JW, Prado R, Chang J, Ginsberg MD, Schleien CL, Busto R: Selective brain cooling increases cortical cerebral blood flow in rats. Am J Physiol Heart Circ Physiol 1993;265: $\mathrm{H} 824-\mathrm{H} 827$.

33 Zhu L: Theoretical evaluation of contribution of heat conduction and countercurrent heat exchange in selective brain cooling in humans. Ann Biomed Eng 2000;28:269-277.

- 34 Wang H, Olivero W, Lanzino G, Elkins W, Rose J, Honings D, Rodde M, Burnham J, Wang D: Rapid and selective cerebral hypothermia achieved using a cooling helmet. J Neurosurg 2004;100:272-277.

35 Kapidere M, Ahiska R, Guler I: A new microcontroller-based human brain hypothermia system. J Med Syst 2005;29:501-512. 